\title{
Personality Dimensions, Smoking Behavior and Drug Effects on Nicotine Dependence: Evidence for Predicting Tobacco Withdrawal
}

\author{
Olimpia Pino $^{1, *}$, Giuliano Giucastro ${ }^{2}$, Annalisa Pelosi $^{1}$ \\ ${ }^{1}$ Department of Neurosciences, University of Parma, Italy \\ ${ }^{2}$ Department of Mental Health and Pathological Dependence, AUSL Langhirano (PR), Italy
}

Copyright $(\subset 2015$ by authors, all rights reserved. Authors agree that this article remains permanently open access under the terms of the Creative Commons Attribution License 4.0 International License

\begin{abstract}
Research on nicotine dependence has had mixed success in identifying variables which can be used to guide treatment and enhance outcome. Personality is one of factors that have been related to the initiation, maintenance and cessation of smoking. This paper aims to analyze relationships between temperamental dimensions, depressive and anxiety symptoms, nicotine dependence and cessation success following different treatment (bupropion $v s$. varenicline). In order to retrospectively investigate the ability of Novelty Seeking (NS), Reward Dependence (RD), Harm Avoidance (HA) and smoking behavior to predict outcomes following pharmacological treatment, we carried out a clinical trial with a total of 162 participants. Subjects are administered with TCI-R, SAS and BDI questionnaires. Nicotine Dependence (ND) and Nicotine Use (CPD) were measured with the Fagerström Test for the Nicotine Dependence (FTND). At post-treatment (3 months) and 12-months follow-up tobacco cessation was measured through self-report and expired air carbon monoxide (CO) test. Results indicated that low level of FTND and Self-Transcendence mildly predicted outcomes. Treatment was not a significant predictor of abstinence. Even if gender not predicted abstinence, women showed a greater difficulty to quit smoking. Findings are discussed in relation to previous studies focusing on theoretical and measurement issues related to dispositional and biological factors.
\end{abstract}

Keywords Nicotine Dependence, Smoking Cessation, Personality Dimensions, Depression, Anxiety

\section{Introduction}

Tobacco use is a risk factor for six of the eight leading causes of death in the world [1] killing one person every 6 sec. and taking away fifteen years of life on average [2]. In the fifth edition of the Diagnostic and Statistical Manual of
Mental Disorder manual [3], the revised chapter of "Substance-Related and Addictive Disorders" combines the DSM-IV categories of substance abuse and substance dependence into a single disorder measured on a continuum from mild to severe. Each specific substance abuse is evaluated as a separate use disorder, but nearly all substances are diagnosed based on the same overarching criteria. Whereas a diagnosis of substance abuse previously required only one symptom, mild substance use disorder in DSM 5 requires two to three symptoms from a list of eleven.

Much recent research has focused on increasing interventions for smoking cessation or defining sub-populations of smokers that may be differentially responsive to treatment. Several studies have found that brief interventions for smoking cessation are efficacious [4-6]. Many findings illustrate that there is significant importance in recognizing personality dysfunctions and styles in treating addiction. A study from the American Journal of Psychiatry revealed that $60 \%$ of subjects with substance use disorders had personality disorders [7]. Others observed that $78 \%$ of the patients interviewed had at least one personality disorder, and the average number of personality disorders was 1.8 per patient [8]; however, no single addictive personality emerged. Most of investigations on the description of trait-like differences between smokers and non-smokers have researched if specific personality traits are involved in Nicotine Dependence (ND). The evidence seems to converge on the importance of impulsivity (Eysenck) as related to substance use [9], sometimes using sensation seeking (Zuckerman) or novelty seeking (Cloninger) conceptualizations [10].

Other research has attempted to associate known biological mechanisms with personality traits[11]. Cloninger proposed a three-dimensional model of personality based on neurogenic adaptive mechanisms [12]. The Temperament and Character Inventory (TCI-R) has four temperament and three character dimensions. The temperament dimensions are Novelty Seeking (NS), Harm Avoidance (HA), Reward 
Dependence (RD), and Persistence (P). The character dimensions are Self-Directedness (SD), Cooperativeness (C), and Self-Transcendence (ST). Character traits serve to diagnose the presence and severity of a disorder, whereas temperament traits indicate differential personality diagnoses. Gaining insights on personality variables of addicted patients provides an opportunity for a more detailed assessment of patient programs and outcomes.

Many studies have used Cloninger's personality model [12-13] and the TCI-R for its predictive value in substance-dependent populations [14-15] showing that high levels of Novelty Seeking predicted higher nicotine use [16-17]. In Nieva et al. [18] investigation, low scores on Sociability predicted high ND and large amount of CPD (number of cigarettes smoked per day) in men and, apart from FTND level (Fagerström Test for the Nicotine Dependence), high levels on Impulsivity predicted relapse in males. One reason for the importance of Impulsivity as evolved trait of temperament and its implications for substance use understanding may be its evolutionary basis: Rhesus monkeys who exhibited novelty-seeking behavior with an unfamiliar object were more likely to drink alcohol [19].

Addressing the elements that sustain tobacco addiction and lead to relapse can enhance successful drugs treatment for tobacco dependence. Nicotine increases dopaminergic activity in areas of the brain associated with reward. Neurotransmitters involved in these processes often are the targets of pharmacologic treatments. Nicotine receptors for Acetylcholine $(\mathrm{ACH})$ are required in order to elicit the reinforcing effects of nicotine. Because dopamine plays a central role in this reinforcing effect, dopaminergic-modulating agents have been considered for the treatment of tobacco abuse. Bupropion increases dopaminergic tone by blocking reuptake of dopamine along with norepinephrine. It prevents or reduces craving and nicotine withdrawal symptoms. However, it produces many side effects and does not work for everyone. The therapeutic action of bupropion also may be related to its noradrenergic effects since a noradrenergic tricyclic antidepressant (nortriptyline) also aids smoking cessation, but serotonin reuptake inhibitors do not [20-21].Subtypes of nicotinic acetylcholine receptors (nAChR) offer the chance to target particular nicotinic actions in the brain. The $\alpha 4 \beta 2$ receptor subtype is the main nicotinic receptor subtype and has a high affinity for nicotine. Considering that the $\alpha 4 \beta 2$ nAChR seems to play an important role in the development of nicotine dependence, various agents selectively targeting this receptor have been developed for testing in humans. Varenicline is used for its high affinity and selectivity at the $\alpha 4 \beta 2$ receptor; it potently increases dopamine release for its agonist effect and blocks the action of nicotine for its antagonist effect. Both bupropion and varenicline seem effective for smoking cessation and have a suppressive effect on symptoms of depression. The present study aimed to investigate differential effects of pharmacotherapy (bupropion vs. varenicline) on smoking cessation in their relationships with temperamental dimensions, anxiety and depressive symptoms. Since approved drug products confer a two-to three-fold increase in quit success rates over placebo, and only about $15-20 \%$ of treatment-seeking smokers are able to remain abstinent after one year, more effective anti-smoking interventions are needed to reduce the prevalence of nicotine abuse. Since bupropion acts regulating the neuroendocrine axis function dopaminergic we anticipated that the effects of bupropion would be different in relationship with NS levels. Cloninger studies, in facts, have highlighted that NS is related with the neuroendocrine dopaminergic axis. Consistent with previous research and theory, we hypothesized that NS and RD would predict cessation outcome (i.e., smoking status within the three-month of a quit attempt and at 12 months follow-up) in participants receiving bupropion treatment. Finally, since there is evidence for major addictive component to heavy smoking, which makes giving up more difficult, we predicted a direct correlation between Nicotine Dependence (ND) or Nicotine Use (CPD) and abstinence probability.

\section{Materials and Methods}

\subsection{Participants}

Participants were recruited for a smoking cessation program settled on the assumption that the interruption efficacy can be enhanced by increasing the frequency of counseling during early stages of the program. Data are collected between 2007 and 2010 at the Antismoking Assistance Integrated Center of the Department of Mental Health \& Pathological Dependence AUSL of Parma (Italy). Medical exclusion criteria included: moderate-severe hypertension ( $\mathrm{PA}>160 / 95 \mathrm{mmHg}$ ), cardiovascular disease or symptoms of significant events (e.g.: angina pectoris, coronary revascularization, diabetes mellitus treated with insulin, myocardial infarction, congestive heart failure, stroke, renal or hepatic impairment or who had received a prescription drug in support of an attempt to quit smoking in the 6 months preceding the start of the program). Psychiatric exclusion criteria included: history of schizophrenia, psychosis, bipolar disorder, disorders of eating behavior, panic attacks, alcohol or drug dependence (past 12 months), and/or hospitalization for psychiatric reasons in the past 12 months. Participants were enrolled if the following inclusion criteria were satisfied: a) range age between 25 and 65 years; b) number of cigarettes smoked per day (CDP) $>10$; c) daily smoking pattern lasting for almost 10 years without considering periods of momentary cessation; d) a score $>$ or $=3$ at the Fagerström Test of Nicotine Dependence (FTND). Individuals who were exclusively cigar or pipe smokers were excluded from the investigation. Women who were pregnant or lactating natural were also ineligible. Finally, the group targeted for study was composed of 162 participants.

\subsection{Measures and Instruments}


At the baseline visit, the clinical history including demographic variables, quitting and smoking history, and current tobacco use were investigated. Fagerström Test of Nicotine Dependence [22] was used as a measure of smoking behavior. This is a standard instrument for assessing the intensity of physical addiction to nicotine. The test (eight items) was designed to provide an ordinal measure of nicotine dependence related to cigarette smoking. The higher the total Fagerström score, the more intense is the patient's physical dependence on nicotine. It predicts smoking abstinence, and resulted correlated with biochemical measures of dependence [23]. Because of a valid measure of addiction can be simply obtained from the number of cigarettes smoked per day (CPD), we used also this value as a measure of nicotine use (NU).

The TCI-R is a self-administered questionnaire [12], which measures seven personality dimensions following a psychobiological and dimensional framework that incorporates the role of neurotransmitters (serotonin, dopamine, and noradrenalin) in behavior regulation. TCI-R scores were converted into T-scores according to published normative data [24]. T-score has a standardized distribution with a mean of 50 and a standard deviation of 10 . The four temperament scales are stable in time, with a genetic base, and measure the adaptation through four psychobiological individual mechanisms.

1. Harm avoidance (HA): Tendency to anxiety, tendency to shyness, worry and avoidance of punishment (hypothetically related to serotonin activity).

2. Novelty seeking (NS): Propensity towards exploratory activity in response to novelty, lack of inhibition, impulsiveness (supposed in relation with the dopaminergic activity) effecting the degree of control or excitability.

3. Reward dependence (RD): Attachment and social attachment systems (hypothetically related to the activity of oxytocin). Associated with attachment to and/or dependence from external approval; being easily influenced; sentimentality/coldness.

4. Persistence (PER): Capability to maintain behavior in harmful conditions (supposed in relation with the noradrenergic activity). Propensity to making demands upon self, hardworking, striving for excellence.

The three character scales are the following:

1. Self-directedness (SD): Maturity, strength and independence. Capacity to manage behavior guided by purposes voluntarily chosen and not by conditions, impulses or external models.

2. Cooperativeness (C): Reveals a disposition towards social tolerance, empathy, friendliness, altruism, and respect for others.

3. Self-transcendence (ST): Reflects a propensity towards spirituality, idealism, religious or mystical feelings and identification with the wider world, as well as the capability to accept ambiguity and uncertainty, and a consciousness of communion with others.
The Zung Self-Rating Anxiety Scale (SAS) was used to quantify anxiety levels. The total raw scores range from 20-80 and needs to be converted into an "Anxiety Index" score for determining the clinical interpretation: 20-44 Normal Range, 45-59 Mild to Moderate Anxiety Levels, 60-74 Marked to Severe Anxiety Levels, 75-80 Extreme Anxiety Levels[25]. Beck Depression Inventory in the 13-item short form (BDI) [26-28] was selected for measuring the severity of depression. Items use a 4-point scale that ranges from zero to 3 . A total score of 8 is the cutoff indicating moderate depression.

\subsection{Procedure}

Participants were assessed at baseline, and at two subsequent times (3 months and 12 months). During the first visit a detailed clinical, psychiatric and smoking history was performed, as for as a clinical examination. Subsequently, questionnaires with measures of personality traits (TCI-R), depressive symptoms (SAS, BDI) and tobacco consumption patterns (FTND) were administered. Cessation motivation was evaluated without the support of psychometric measures. All participants gave written informed consent, and the confidentiality for personal data and study results was fully guaranteed. Carbon monoxide (CO) was used as a measure of tobacco exposure. A value greater than eight parts per million obtained from the measurement of exhaled $\mathrm{CO}$ is the criterion standard for the diagnosis of active smoking status [29]. We analyzed participants' breath samples using a Bedfont piCO Smokerlyzer - Bedfont Scientific Ltd.

Then they were assigned to receive bupropion $(\mathrm{n}=101)$, varenicline $(\mathrm{n}=46)$ intervention programs or counseling $(n=15)$ as control. During the first visit, for participants of all groups, a quit date within a two-week window was also stated. Because of certain individual characteristics affect a person's possibility of success in stopping smoking, and many of these characteristics can be adapted in order to increase the success likelihood, additional suggestions on coping strategies in the manage of stopping or health consequences were dispensed.

A second control visit was planned two weeks later coinciding with the $24 \mathrm{~h}$ of total smoking cessation with the purpose to verify both patients' compliance with clinical suggestions and successful cessation (post-treatment session). Particular attention was devoted to the expired $\mathrm{CO}$ confirmation of patient self-reports for abstinence. Because of pharmacological therapy due to the economic burden was patient-dependent, there was a lack of uniformity within participants for the duration of the treatment that was extended to approximately a month and half (standard dose was of $150 \mathrm{mg}$ and $1 \mathrm{mg}$ on a twice daily basis for bupropion and varenicline, respectively). The counseling treatment consists of approximately 3 sessions of 30 minutes in duration for the in-person and telephone modalities. Counseling involved active effort to identification and control of high-risk situations for smoking (using functional analysis approach for identifying antecedences and 
consequences of smoking behavior and relevant thoughts), development of coping skills based on the individual level of knowledge base and motivation, and direct support after the quit date in order to use adaptive strategies.

During post-treatment and follow-up sessions, dropouts were evaluated as relapses and the others, if confirmed by CO measure, as clinically abstinent.

\subsection{Data Analysis}

In order to investigate personality traits and other variables as predictors of smoking cessation after 3 months of treatment and 12 month follow-up, a series of logistic regression analyses using the stepwise method was conducted. The purpose was to examine the associations between hypothesized predictors (personality traits, depressive symptoms, age, gender, medical treatment) with dichotomous (male vs. female) and dimensional (age) variables, and smoking outcome (abstainers vs. relapses) at two different time periods (3 and 12 months). In order to evaluate gender effects on dependent variables as accessories hypotheses, analyses of variance (AN.O.VA.) were also used. All effects were tested at a two-tailed alpha of 0.05 and performed using SPSS (15.0; C SPSS Inc., 2006). Logistic regression and Cox proportional hazards regression were used to evaluate our hypotheses. Demographic variables (age and gender), smoking status (FTND and CPD), personality dimensions (NS, HA, RD, P, SD, C and ST), depressive and anxiety symptoms, and medical treatment were entered as predictors in each analysis. For all cases considered, we estimated the likelihood of being abstainers vs. smokers at three and twelve months following treatment. The odds ratio (OR) were calculated to estimate the likelihood of being abstainers as compared with smokers. Two tailed $95 \%$ CI were also computed.

The models were tested against the global null hypothesis using the log likelihood ratio test. Their goodness of fit was tested using the Hosmer and Lemeshow [29] test. Differences in proportions of categorical variables were tested for significance by a chi-square $\left(\chi^{2}\right)$ test.

\section{Results}

In Figure 1.percent of abstainers for the three groups at post-treatment and follow-up (3 and 12 months) measurements is depicted.

Table 1. presents mean and standard deviation for each group and total sample in all measures. In Table 2 logistic regression model was reported. The mean age of participants (Table 1) included in the present study was of 45.7 years $(\mathrm{SD}=10.2)$. Their mean FTND score for the total sample was $6.3(\mathrm{SD}=2.1)$, the mean tobacco consumption was of 23.1 cigarettes per day (CPD) $(\mathrm{SD}=9.5)$, and the value of $\mathrm{CO}_{2}$ expired of $22.8(\mathrm{SD}=10.6)$.

No significant difference between groups resulted at baseline (univariate ANOVA) both in TCI dimensions and Zung and BDI scores. The counseling group, however, was older $\left(\mathrm{F}_{2,159}\right)=4.94, \mathrm{p}<.01, \eta^{2}=.06$; Tukey's post hoc test: $\mathrm{p}<.05)$ and showed a significant prevalence of the low dependence category at Fagerstrom test $(\leq 6$; standardized cell residuals $Z=2.3, \mathrm{p}<.05$ ).

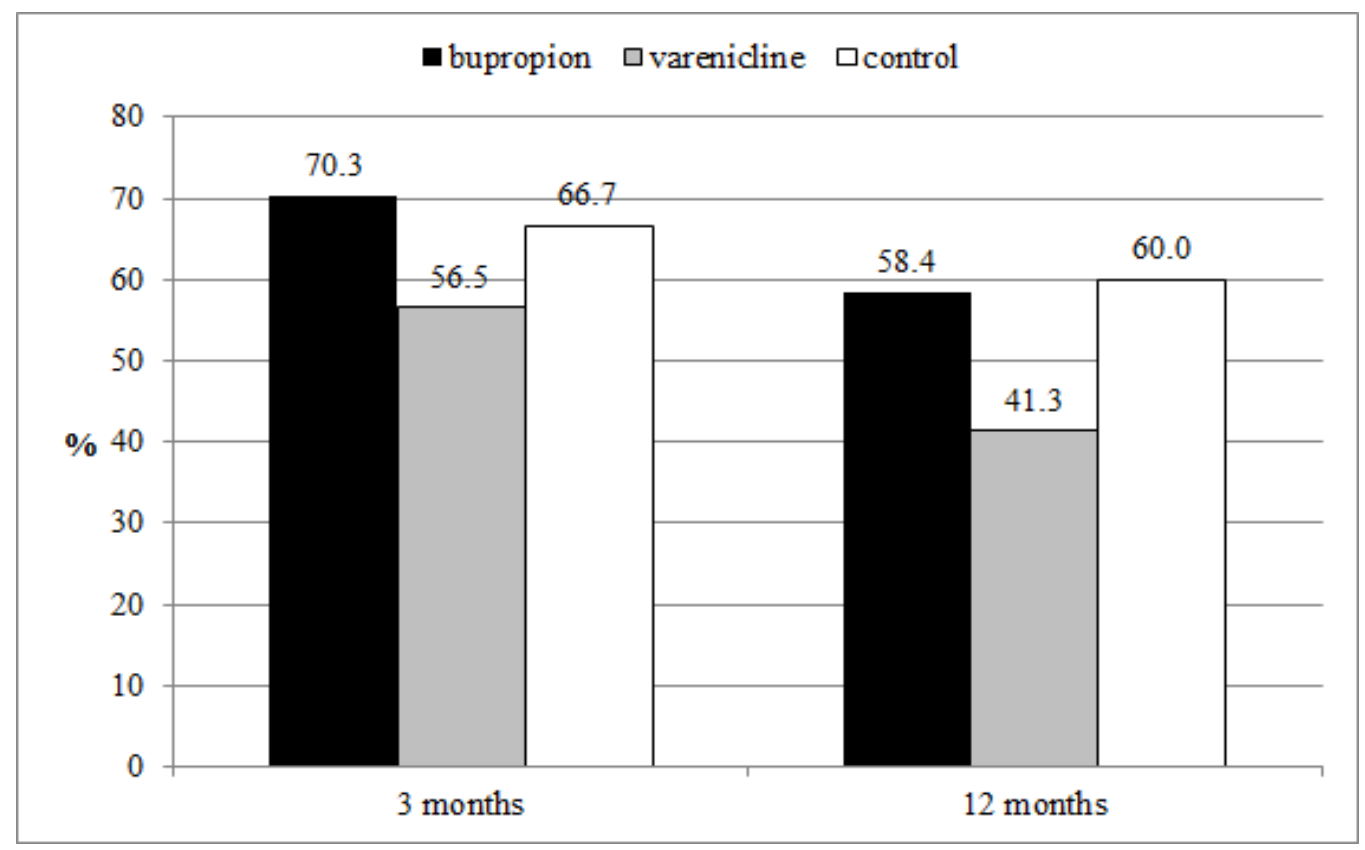

Figure 1. Percent of abstainers for the three groups at post-treatment and follow-up ( 3 and 12 months) measures 
Table 1. Groups means (standard deviation)

\begin{tabular}{|c|c|c|c|c|c|c|c|c|c|}
\hline & & \multicolumn{2}{|c|}{ Total Sample } & \multicolumn{2}{|c|}{ Bupropion $(\mathrm{N}=101)$} & \multicolumn{2}{|c|}{ Varenicline $(\mathrm{N}=46)$} & \multicolumn{2}{|c|}{ Counseling ( $N=15)$} \\
\hline Age & & \multicolumn{2}{|c|}{$45.7(10.2)$} & \multicolumn{2}{|c|}{$45.33(9.2)$} & \multicolumn{2}{|c|}{$44.07(11.45)$} & \multicolumn{2}{|c|}{$53.2(10.1)$} \\
\hline \multirow{7}{*}{ TCI } & NS & \multicolumn{2}{|c|}{$48.1(14.4)$} & \multicolumn{2}{|c|}{$49.1(15.2)$} & \multicolumn{2}{|c|}{$46.9(12.7)$} & \multicolumn{2}{|c|}{$45.0(14.0)$} \\
\hline & HA & \multicolumn{2}{|c|}{$48.8(18.8)$} & \multicolumn{2}{|c|}{$48.4(18.8)$} & \multicolumn{2}{|c|}{$48.5(19.4)$} & \multicolumn{2}{|c|}{$52.6(17.1)$} \\
\hline & $\mathrm{RD}$ & \multicolumn{2}{|c|}{$61.5(14.9)$} & \multicolumn{2}{|c|}{$61.4(15.3)$} & \multicolumn{2}{|c|}{$62.0(15.3)$} & \multicolumn{2}{|c|}{$61.4(11.2)$} \\
\hline & $\mathrm{P}$ & \multicolumn{2}{|c|}{$57.2(24.7)$} & \multicolumn{2}{|c|}{$59.3(22.9)$} & \multicolumn{2}{|c|}{$53.3(28.8)$} & \multicolumn{2}{|c|}{$55.0(23.0)$} \\
\hline & SD & \multicolumn{2}{|c|}{$66.5(16.8)$} & \multicolumn{2}{|c|}{$66.5(16.6)$} & \multicolumn{2}{|c|}{$63.9(18.3)$} & \multicolumn{2}{|c|}{$73.9(11.5)$} \\
\hline & & 73.6 & 14.2) & 72.8 & 4.7) & 73.8 & 3.8) & 78.4 & 1.7) \\
\hline & & 38.3 & 16.9) & 37.6 & 7.5) & 41.1 & $6.4)$ & 34.1 & 3.5) \\
\hline & & 6.3 & & & & & & & \\
\hline & & $M$ & $F$ & $M(N=57)$ & $F(N=44)$ & $M(N=24)$ & $F(N=22)$ & $M(N=8)$ & $F(N=7)$ \\
\hline $\mathrm{CO}^{2}$ & & $23.1(10.8)$ & $22.4(10.4)$ & $24.2(9.1)$ & $24.8(9.5)$ & $23.6(14.2)$ & $20.3(10.9)$ & $13.5(6.2)$ & $14.3(9.8)$ \\
\hline & Total & 22.8 & 10.6) & 24.4 & & 22.1 & (2.7) & 13.9 & 7.8) \\
\hline & & $24.9(10.5)$ & $20.9(7.6)$ & $25.9(11.7)$ & $22.8(8.3)$ & $24.3(6)$. & $19.7(3.9)$ & $18.6(9.1)$ & $12.9(6.3)$ \\
\hline & Total & 23.1 & 9.5) & 24.6 & $0.5)$ & 22. & 6.1) & 15.9 & 8.2) \\
\hline & & Total & ample & Bupropi & $(\mathrm{N}=76)$ & Varenic & e $(N=37)$ & Counseli & $(\mathrm{N}=13)$ \\
\hline & re) & 32.0 & 8.4) & 32.3 & 3.0) & & 9.8) & 29.5 & 6.3) \\
\hline & & 40.3 & 10.4) & 40.5 & $0.1)$ & 40.8 & 1.8) & 37.7 & 7.8) \\
\hline & & 5.2 & 3.8) & 5.2 & & & 1.9) & 5.0 & .2) \\
\hline
\end{tabular}

Table 2. Logistic regression model

\begin{tabular}{|c|c|c|c|c|c|c|c|}
\hline & \multirow[b]{2}{*}{ Outcome } & \multicolumn{2}{|c|}{ Model fitting } & \multicolumn{4}{|c|}{ Predictors } \\
\hline & & $\begin{array}{l}\text { Likelihood } \\
\text { Ratio Test }\end{array}$ & Nagelkerke $\mathbf{R}^{2}$ & & B (SE) & Wald test $\chi_{2}^{2}$ & OR (95\%IC) \\
\hline & \multirow{2}{*}{ Three m. } & \multirow{2}{*}{$\begin{array}{c}\chi_{2}^{2}=15.71 \\
\mathrm{p}<.01\end{array}$} & \multirow{2}{*}{.166} & FTND & $-.364(.01)$ & 10.45. $\mathrm{p}<.01$ & $.695(.557-.866)$ \\
\hline \multirow{3}{*}{ Total sample } & & & & ST & $-.023(.11)$ & 3.86. $\mathrm{p}<.05$ & $.977(.955-1.0)$ \\
\hline & \multirow{2}{*}{ Twelve m. } & \multirow{2}{*}{$\begin{array}{c}\chi_{2}^{2}=15.82 \\
\mathrm{p}<.01\end{array}$} & \multirow{2}{*}{.161} & FTND & $-.215(.01)$ & 4.75. $\mathrm{p}<.05$ & $.807(.665-.979)$ \\
\hline & & & & ST & $-.037(.1)$ & 10.24. $\mathrm{p}<.01$ & $.963(.942-.986)$ \\
\hline \multirow{2}{*}{ Buproprion } & Three m. & $\chi_{2}^{2}=6.07 . p<.05$ & .113 & FTND & $-.37(.16)$ & 5.41. $\mathrm{p}<.05$ & $.691(.506-.944)$ \\
\hline & Twelve $\mathrm{m}$. & -- & -- & -- & -- & -- & -- \\
\hline \multirow{5}{*}{ Varenicline } & \multirow{3}{*}{ Three m. } & \multirow{3}{*}{$\begin{array}{c}\chi_{2}^{2}=17.42 \\
p<.01\end{array}$} & \multirow{3}{*}{.507} & FTND & $-.794(.35)$ & 5.06. $\mathrm{p}<.05$ & $.452(.226-.903)$ \\
\hline & & & & ST & $-.077(.03)$ & $5.53 \mathrm{p}<.05$ & $.926(.869-.987)$ \\
\hline & & & & $\mathrm{P}$ & $-.034(.02)$ & 4.51. $\mathrm{p}<.05$ & $.967(.937-.997)$ \\
\hline & \multirow{2}{*}{ Twelve m. } & \multirow{2}{*}{$\begin{array}{c}\chi_{2}^{2}=14.69 \\
p<.01\end{array}$} & \multirow{2}{*}{.438} & ST & $-.087(.03)$ & $8.16 . \mathrm{p}<.01$ & $.917(.864-.973)$ \\
\hline & & & & $\mathrm{P}$ & $-.029(.01)$ & 3.74. $\mathrm{p}<.05$ & $.971(.943-1.0)$ \\
\hline \multirow{2}{*}{ Counseling } & Three m. & $\chi_{2}^{2}=7.79 . p<.01$ & .66 & ST & $-.221(.13)$ & 2.81. $\mathrm{p}<.05$ & $.801(.618-.984)$ \\
\hline & Twelve $\mathrm{m}$. & $\chi_{2}^{2}=5.44 . p<.05$ & .491 & ST & $-.14(.08)$ & 2.86. $\mathrm{p}<.05$ & $.869(.739-.998)$ \\
\hline
\end{tabular}

Following treatment (Figure 1) the $70.3 \%$ of participants who received bupropion lapsed within 3 months and only the $58.4 \%$ remain abstinent at the 12 months follow-up while the $56.5 \%$ of subjects treated with varenicline and the $66.7 \%$ in counseling group becomes abstinent at 3 months and the $41.3 \%$ and the $60 \%$ at 12 months, respectively. In order to examine the predictive relationship between the considered variables, a forward logistic regression was run, evaluating gender, age, TCI dimensions, Zung and BDI scores, Fagerstrom score and therapy effects on the outcomes (abstinent versus not abstinent) at three months post-treatment (T1) and twelve months (T2) follow-up. As regard the total sample (Table 2), at $\mathrm{T} 1$ evaluation only the
Fagerstrom score and, less strongly, the TCI Self -Trascendence (ST) determined a mildly predictive, even significant, model: a greater probability to be abstinent was related to a lower baseline nicotine dependence and to a lower ST. Both dimensions maintained their predictive capability until T2, though ST slightly increased its prevalence into the model. Fagerstrom score and ST confirmed their effects on the outcome along the three therapy groups, in spite of some small distinctiveness. In buproprion group, only the Fagerstrom score revealed a mild effect on theT1 outcome: the less dependent patients had a greater probability to be abstinent; no variable, however, was significant at $\mathrm{T} 2$. A positive outcome at $\mathrm{T} 1$ for the patients 
treated with varenicline was instead determined, besidesby lower Fagerstrom and ST scores, also by lesser Persistence; only the Persistence (but its statistical significance was only at threshold) and Self Transcendence scores remained significantly predictive at T2. At last, the counseling patients' outcomes were only influenced by ST; both at $\mathrm{T} 1$ and at $\mathrm{T} 2$, the Fagerstrom score did not satisfied the statistical $p$-threshold criterion $(\mathrm{p}=.07)$ and, consequently, was not introduced into the prediction model.

Confirming the logistic regression suggestions, no differences emerged between smokers and no smokers in Zung and BDI scores, both at $\mathrm{T} 1$ and at $\mathrm{T} 2$, independently from the therapy (bivariate ANOVAs Group x Outcome). The temperamental and character traits were no different between smokers and no smokers at $\mathrm{T} 1$, even if in the counseling group the smokers showed higher self-transcendence $\left(46.1 \pm 12.4\right.$ versus $28.2 \pm 9.9 ; \mathrm{F}_{[1,13]}=9.26$, $\left.\mathrm{p}<.01 . \eta^{2}=.41\right)$. The ST proved itself as more stronger among the smokers at $\mathrm{T} 2$, less clearly in the buproprion group (41.1 \pm 14.9 vs $\left.35.1 \pm 18.8 ; \mathrm{F}_{[1,99]}=3.04, \mathrm{p}<.06, \eta^{2}=.02\right)$, but more evidently in the varenicline $(47.4 \pm 13.9$ vs $\left.32.2 \pm 15.8 ; \mathrm{F}_{[1,44]}=11.87, \mathrm{p}<.01, \eta^{2}=.21\right)$ and counseling $\left(42.9 \pm 13.5 v s \quad 28.3 \pm 10.5 ; \quad \mathrm{F}_{[1,13]}=5.61, \quad \mathrm{p}<.05, \quad \eta^{2}=.31\right)$ groups.

The type of treatment, therefore, was not a significant predictor for the abstinence, neither for a short-term nor for a long-term follow up. At T1, no association between treatment and outcome emerged ( $\chi^{2}$ test). At T2, the slight prevalence of no smokers in buproprion and counseling groups did not hit the statistical significance (standardized cell residuals, $p>.05$ ); but the prevalence of smokers in varenicline groups was significantly greater than chance $(\mathrm{Z}=$ $2.1, \mathrm{p}<.05)$.

The consistency between $\mathrm{T} 1$ and $\mathrm{T} 2$ outcomes was remarkable $\left(\chi^{2}=91.23, p<.01\right.$, contingence index $\mathrm{C}=.599$, $\mathrm{OR}=225.24)$. In the total sample, the $98.2 \%$ of the subjects who smoked at $\mathrm{T} 1$ did not have changed habits at T2 (standardized cell residuals $Z=9.6 . p<.01$ ); among the abstainers at $\mathrm{T} 1$, the $19.6 \%$ fall again into the nicotine abuse at T2. Considering the abstainers as target category, then, the odds decreased from 1.91 three months after the baseline to 1.14 after a year. In buproprion group $\left(\chi^{2}=53.31, p<.05\right)$, the $96.7 \%$ of smokers at $\mathrm{T} 1$ did not change at $\mathrm{T} 2(\mathrm{Z}=7.3, \mathrm{p}<.01)$, whereas the $18.3 \%$ of abstainers fell back into dependence (maintenance: $81.7 \%)$. In varenicline group $\left(\chi^{2}=24.9\right.$. $\left.p<.05\right)$ no one of the twenty smokers at $\mathrm{T} 1$ has become abstinent at $\mathrm{T} 2(\mathrm{Z}=5.1 . \mathrm{p}<.01)$, and the $26.9 \%$ ofT1 abstainers relapsed into the dependence after a year (maintenance: $73.1 \%$ ). At last, among the counseling group $\left(\chi^{2}=11.2 . p<.05\right)$ no smokers at T1 (five subjects) stopped smoking, and the 10\% of abstainers had restarted at T2 (maintenance: $90 \%$ ).

Even if gender was not a significant outcome predictor, some clinical implications can be derived from the evidence that women, independently from treatment programs, showed a greater difficulty to quit smoking. The percentage of female abstainers was lower at $\mathrm{T} 1(58.9 \%$ vs. $71.1 \%$; $\mathrm{Z}=-1.6, \mathrm{OR}=.582)$ and, mainly, at $\mathrm{T} 2(45.2 \%$ vs. $60.0 \%$;
$\mathrm{Z}=-1.9, \mathrm{OR}=.550$ ), even if no significant difference between genders resulted in Self-Trascendence, Persistence and FTND (univariate ANOVAs).

\section{Discussion and Conclusions}

The first aim of the present study was to analyze whether the temperamental dimensions, depressive and anxiety scores are influential in the achievement of abstinence and if their effect are in relationships with different treatments. Since bupropion acts by regulating the neuroendocrine axis function dopaminergic, we anticipated that its influence would be different in individuals with different levels of Novelty Seeking. Our findings are unable to find support for the first hypothesis: Novelty Seeking (NS), Reward Dependence (RD) and Harm Avoidance not predict smoking cessation outcome, and we did not find any relationship with bupropion. Thus, we were unable to replicate findings from previous studies [31]. RD also was not resulted in relationship with tobacco abuse.

As Brown et al. [32] argue, as a predictor of smoking cessation outcome, Persistence may be especially important to assess in presence of stressors. In their perspective, smokers who relapse soon after their quit day often do so because they have great difficulty tolerating the stress of nicotine withdrawal symptoms [33]. In our trial Persistence revealed an effect on abstinence only for participants who received varenicline and a low level of Self-Transcendence predicted outcome. No differences emerged between smokers and abstainers in depression and anxiety scores, but their level were in the normal range. For consequence, we were unable to evaluate the effect of treatment on these characteristics. The perspective that certain PDs on Axis II disorders (borderline, antisocial, schizoid, and avoidant) can predict treatment results influencing at different times of the cessation trajectory, such initial cessation and maintenance of abstinence [34] in our study is impossible to verify. Previous investigations found that smokers have higher rates of PDs, although these rates are very variable [35-36].Although the anti-tobacco properties of bupropion appear to be unrelated to their antidepressant activity, they may benefit persons with low drive and low positive affect. However, bupropion may be damaging for individuals with cyclothymic, dysphoric or irritable temperaments, since it can increase impulsivity, mood instability, and induce suicidal ideation [37]. Our findings failed to show any influence on outcome due to the relationship between pharmacotherapy, depression and anxiety scores. Since, our participants showed depression and anxiety scores in the normal range, but it would have useful to use irritability scale and mood disorder questionnaire in order to capture the entire spectrum of mood and anxiety symptoms. Further investigations are necessary for clarifying the relationships between pharmacotherapies, PD and smoking.

Our study has some strengths, including the use of a sample of community-based participants, and its sample size. A limitation is that some information about the smoking 
phenotypes, such as the age of onset was not assessed. For that reason, we limited our investigation to variables that we considered particularly salient. However, neurobiological and genetic theories of substance dependence are mixed, with respect to whether aspects of addiction are the result of preexisting characteristics or damage to key neural circuitry associated with nicotine dependence. Future research should also consider factors that may moderate the relationship on distress tolerance involved in quitting smoking. The initial tobacco use may be influenced by a variety of factors, including availability and the rewarding properties of its use, but tobacco addiction represents an inability to control use one it has begun. Many data support the idea that different factors predispose individuals to initial versus continued use of tobacco. Targets for the treatment of smoking behavior can include decreasing the reinforcing effects of tobacco and decreasing the effects of triggers on repeated use or relapse, such as environmental cues, stress, or negative emotions, which work through different neurobiological pathways.

Pharmacotherapies in our trial do not represent a predictive factor for smoking cessation. The effect of medical treatment can be implicated from a large number of biological functions and regions (e.g. involving dopaminergic function and nicotine metabolism). The therapeutic action of bubropion may be related to its noradrenergic effects because a noradrenergic tricyclic antidepressant also aid smoking quitting, but serotonin reuptake inhibitors do not. In addition to its other actions, bupropion functions as antagonist at the nicotine acetylcholine receptors $(\mathrm{nAChR})$ and may block the rewarding effects of nicotine through his mechanism. Bupropion is a dopamine/norepinephrine reuptake inhibitor that also acts as a nicotinic receptor antagonist [38]; varenicline is a partial agonist of the $\alpha 4 \beta 2 \mathrm{nAChR}$ subtype [39].

There are many evidences that the modulation of nicotine withdrawal symptoms may contribute to their effectiveness. Our findings do not suggest that bupropion and varenicline may actually be associated with a generalized suppression of some symptoms of depression during cessation, and have no impact on anxiety. The absence of clinical levels of depression and anxiety symptoms reduce this probability. Our study suggested that only the intensity of nicotine dependence was a significant predictor of abstinence. The determinants of successful smoking cessation, like nicotine addiction itself, are likely to be diverse and recent investigation [40]indicated that multiple genetic loci are associated with varenicline $v s$. bupropion response, suggesting that additional research may identify clinically useful markers to guide treatment decisions.

\section{Contributors}

All the authors designed the study and wrote the protocol.
All authors conducted literature searches and provided summaries of previous research studies. AP conducted the statistical analysis. GG and OP wrote the first draft of the manuscript and all authors contributed to and have approved the final version.

\section{Conflict of Interest}

All authors declare that they have no conflicts of interest.

\section{REFERENCES}

[1] World Health Organization. The world health report 2003: Shaping the future. Geneva, Switzerland: World Health Organization, 2003.

[2] World Health Organization. Report on the global tobacco epidemic, 2008. The MPOWER package. Geneva, Switzerland: World Health Organization, 2008.

[3] American Psychiatric Association. Diagnostic and statistical manual of mental disorders (5th Ed.). Washington, DC: Author, 2013.

[4] M.C. Fiore et al. Treating tobacco use and dependence: 2008 Update. Clinical Practice Guideline. U.S. Department of Health and Human Services, Public Health Service, 2008.

[5] S.S. Gorin\& J.E. Heck. Meta-analysis of the efficacy of tobacco counseling by health care providers. Cancer Epidemiology Biomarkers and Prevention, 13, 2012-2022, 2004.

[6] Sistema Nazionale per le Linee Guida dell'Istituto Superiore di Sanità (SNLG-ISS). Prevenzione primaria del fumo di tabacco. Linee guida di prevenzione. Milano, Italia: Geca, 2013.

[7] A.E. Skodol, J.M. Oldham \& P.E. Gallaher. Axis II comorbidity of substance use disorders among patients referred for treatment of personality disorders. American Journal of Psychiatry, 156, 733-738, 1999.

[8] C.A. De Jong, W. van den Brink, F.M. Harteveld, \& E.G. van der Wielen. Personality disorders in alcoholics and drug addicts. Comprehensive Psychiatry, 34, 87-94, 1993.

[9] M.N. Sargeant, M.A. Bornovalova, A.J. Trotman, S. Fishman, \& C.W. Lejuez. Facets of impulsivity in the relationship between antisocial personality and abstinence. Addictive Behaviors, 37, 293-298, 2012. Avalaible from: http://dx.doi.org/10.1016/j.addbeh.2011.11.012.

[10] M. Zuckerman \& C.R. Cloninger Relationships between Cloninger's, Zuckerman's, and Eysenck's dimensions of personality. Personality and Individual Differences, 21, 283-285, 1996

[11] S.G. Acton. Measurement of impulsivity in a hierarchical model of personality traits: Implications for substance use. Substance Use \& Misuse, 38, 67-83, 2003.

[12] C.R. Cloninger, D.M. Svrakic\& T.R. Przybeck. A psychobiological model of temperament and character. 
Archives of General Psychiatry, 50, 975-990, 1993.

[13] C.R. Cloninger. A systematic method for clinical description and classification of personality variants: A proposal. Archives of General Psychiatry, 44, 573-588, 1986.

[14] D. Kalman, R. Hoskinson, U. Sambamoorthi, \& A.J. Garvey. A prospective study of persistence in the prediction of smoking cessation outcome: Results from a randomized clinical trial. Addictive Behaviors, 35, 179-182, 2010. doi:10.1016/j.addbeh.2009.09.017.

[15] C.S. Pomerleau, O.F. Pomerleau, K.A. Flessland\& S.M. Basson. Relationship of Tridimensional Personality Questionnaire scores and smoking variables in female and male smokers. Journal of Substance Abuse, 4, 143-154, 1992.

[16] M. Gurpegui, D. Jurado, J.D. Luna, C. Fernández-Molina, O. Moreno-Abril \& R. Gálvez. Personality traits associated with caffeine intake and smoking. Progress in Neuro-Psychopharmacology \& Biological Psychiatry, 31, 997-1005, 2007.

[17] M. Zuckerman, S. Ball \& J. Black. Influences of sensation seeking, gender, risk appraisal, and situational motivation on smoking. Addictive Behaviors, 15, 209-220, 1990.

[18] G. Nieva et al. The alternative five-factor model of personality, nicotine dependence and relapse after treatment for smoking cessation. Addictive Behaviors, 36, 965-971, 2011. doi:10.1016/j.addbeh.2011.05.008.

[19] S.J. Suomi. Behavioral inhibition and impulsive aggressiveness: insights from studies with Rhesus monkeys. In L. Balter\& C.S. Tamis-LeMonda (Eds.) Child psychology: A handbook of contemporary issues. Philadelphia: Taylor \& Francis, 510-525, 1999.

[20] S.G. Gourlay, L.F. Stead \& N.L. Benowitz. Clonidine for smoking cessation. Cochrane Database of Systematic Reviews, CD000058.pub2. 2004. doi: 10.1002/14651858.

[21] D.S. Harris \& R.M. Anthenelli. Expanding treatment of tobacco dependence. Current Psychiatry Reports, 7, 344-351, 2005.

[22] T.F. Heatherington, L.T., Kozlowski, R.C. Frecker\& K.O. Fagerström. The Fagerström Test for Nicotine Dependence: A revision of the Fagerström Tolerance Questionnaire. British Journal of Addiction, 84, 791-800, 1991.

[23] M. Pérez-Rìos, M.I. Santiago-Pérez, B. Alonso, A. Malvar, X. Hervada \& J. de Leon. Fagerstrom test for nicotine dependance vs. heavy smoking index in a general population survey. BMC Public Health, 9, 493, 2009. doi: 10.1186/1471-2458-9-493.

[24] C.R. Cloninger et al. The Temperament and Character Inventory (TCI): A Guide to Its Development and Use. St Louis, MO: Center for Psychobiology of Personality, 1994.

[25] W.W.K. Zung. A rating instrument for anxiety disorders. Psychosomatics, 12, 371-379, 1971. doi: 10.1016/S0033-3182(71)71479-0.

[26] A.T. Beck, C. Ward \& M. Mendelson. Beck Depression Inventory (BDI). Archives of General Psychiatry, 4, 561-571,
1961. doi:10.1001/archpsyc.1961.01710120031004.

[27] A.T. Beck, R.A. Steer \& M.G.J. Garbin, Psychometric properties of the Beck Depression Inventory Twenty-five years of evaluation. Clinical Psychological Review, 8, 77-100, 1988. doi:10.1016/0272-7358(88)90050-5.

[28] G. Groth-Marnat. The handbook of psychological assessment (2nd Ed.). New York: John Wiley \& Sons, 1990.

[29] L. Marino et al. Definition of a reliable threshold value for detecting current smokers by $\mathrm{CO}$ measurement: preliminary results. Communication presented at The Society for Research on Nicotine and Tobacco. Second European Conference on Tobacco Addiction. London: England November 25th-26th, pp. 59, 1999.

[30] D.W. Hosmer \& S. Lemeshow. Applied logistic regression. New York: Wiley, 1989.

[31] J.D. Flory \& S.B. Manuck. Impulsiveness and cigarette smoking. Psychosomatic Medicine, 71, 431-437, 2009.

[32] R.A. Brown, C.W. Lejuez, C.W. Kahler, D.R. Strong \& M.J. Zvolensky. Distress tolerance and early smoking lapse. Clinical Psychology Review, 25, 713-733, 2005.

[33] T.B. Baker, M.E. Piper, D.E. McCarthy, M.R. Majeski\& M.C. Fiore. Addiction motivation reformulated: an affective processing model of negative reinforcement. Psychological Review, 111, 33-51, 2004.

[34] B. Piñeiro, E. Fernándezdel Río, A. López-Durán, U. Martínez, \& E. Becoña. The association between probable personality disorders and smoking cessation and maintenance. Addictive Behaviors, 38, 2369-2373, 2013. Online available from http://dx.doi.org/10.1016/j.addbeh.2013.03.017.

[35] P. Moran, C. Coffey, A. Mann, J.B. Carlin \& G.C. Patton. Personality and substance use disorders in young adults. The British Journal of Psychiatry, 188, 374-379, 2006. Online available from http://dx.doi.org/10.1192/bjp.188.4.374.

[36] T.J. Trull, S. Jahng, R.L. Tomko, P.K. Wood \& K.J. Sher. Revised NESARC personality disorder diagnoses: Gender, prevalence, and comorbidity with substance dependence disorders. Journal of Personality Disorders, 24, 412-426, 2010. Online available from http://dx.doi.org/10.1521/pedi.2010.24.4.412.

[37] H.S. Akiskal et al. Validating antidepressant-associated hypomania (bipolar III): A systematic comparison with spontaneous hypomania (bipolar II). Journal of Affective Disorders, 73, 65-74, 2003.

[38] C. Warner \& M. Shoaib. How does bupropion work as a smoking cessation aid? Addiction Biology, 10, 219-231, 2005.

[39] J.W. Coe, et al. Varenicline: An alpha4beta2 nicotinic receptor partial agonist for smoking cessation. Journal of Medical Chemistry, 48, 3474-3477, 2005.

[40] King, D.P. et al. Smoking cessation pharmacogenetics: Analysis of varenicline and bupropion in placebo-controlled clinical trials. Neuropsychopharmacology, 37, 641-650, 2012. doi:10.1038/npp.2011.232. 